\title{
DIMENSION AND INFINITE PRODUCTS IN SEPARABLE METRIC SPACES
}

\author{
JOHN KULESZA
}

(Communicated by Dennis Burke)

ABSTRACT. For each pair $n, d$ of positive integers with $n \leq d$, there is a separable metric space $X_{n d}$ satisfying $\operatorname{dim}\left(X_{n d}\right)=n$ and $\operatorname{dim}\left(X_{n d}^{\omega}\right)=d$.

\section{INTRODUCTION}

One of the most interesting parts of the dimension theory of separable metric spaces is the behavior of the dimension function with respect to products. While one expects, for nice spaces $X$ and $Y$, that $\operatorname{dim}(X \times Y)=\operatorname{dim}(X)+\operatorname{dim}(Y)$, it turns out that this "logarithmic" law fails even for compact metric spaces. (The first such example is due to Pontrjagin [Po].) However, the following theorem does hold for separable metric spaces.

Theorem 1. If $0<\operatorname{dim}(A), \operatorname{dim}(B)<\infty$, and either $A$ or $B$ is compact, then $\operatorname{dim}(A \times B)>\max \{\operatorname{dim}(A), \operatorname{dim}(B)\}$.

In 1967, in [AK], Anderson and Keisler showed that the compactness condition could not be removed completely by giving, for each $n \in \omega$, a separable metric space $X_{n}$ satisfying $\operatorname{dim}\left(X_{n}\right)=\operatorname{dim}\left(X_{n}^{2}\right)=\operatorname{dim}\left(X_{n}^{\omega}\right)=n$. More recently, the author has shown, in [K], that spaces with the properties of $X_{n}$ can be chosen which are additionally topologically complete; hence the compactness condition of Theorem 1 cannot be weakened. Interestingly, these spaces never become higher dimensional upon taking products, even infinite products. The proof relies on the following theorem (which is actually more general, see [P] and $[\mathrm{AK}])$.

Theorem 2. If $A$ is a metric space, then $\operatorname{dim}\left(A^{\omega}\right) \leq k$ if and only if, for all $n \in \omega, \operatorname{dim}\left(A^{n}\right) \leq k$.

All of this leads one to wonder whether a space $X$ can have its infinite powers with higher dimension than $X$ but still be finite dimensional. It follows from Theorem 1 that such a space cannot be compact. In this paper we prove the following theorem.

Received by the editors May 22, 1989 and, in revised form, September 15, 1989.

1980 Mathematics Subject Classification (1985 Revision). Primary 54F45, 54G20.

Key words and phrases. Separable metric space, dimension, product space, general position. 
Theorem 3. Let $n$ and $d$ be integers with $0<n \leq d$. Then there is a separable metric space $X_{n d}$ satisfying $\operatorname{dim}\left(X_{n d}\right)=n$ while $\operatorname{dim}\left(X_{n d}^{\omega}\right)=d$.

The examples above work for the special case $n=d$. Note that, by Theorem 2 , we will only have to consider the dimension of finite products.

In terms of techniques, we keep the dimension down by generalizing the Anderson and Keisler technique; we get the dimension to go up using ideas in [RSW].

\section{Preliminaries}

Let $\mathbf{R}$ denote the real numbers and let $\mathbf{I}$ denote the closed interval $[0,1]$. We will consider $x \in \mathbf{R}^{n}$ or $\left(\mathbf{I}^{n}\right)$ as $x=\left(x_{0}, x_{1}, \ldots, x_{n-1}\right)$. If $A$ and $B$ are disjoint closed subsets of a space $X$, then a closed set $S$ is a separator of $(A, B)$ provided $X-S$ is a union of two disjoint open sets, one containing $A$ and the other containing $B$. If $\left\{\left(A_{k}, B_{k}\right): k \in T\right\}$ is a collection of pairs of disjoint closed sets in $X$ such that whenever $S_{k}$ is a separator of $\left(A_{k} B_{k}\right)$ in $X$ it follows that $\bigcap_{k \in T} S_{k} \neq \varnothing$, we say $\left\{\left(A_{k}, B_{k}\right): k \in T\right\}$ is an essential family for $X$. The standard essential family for $\mathbf{I}^{n}$ is $\left\{\left(A_{k}, B_{k}\right): k \in n\right\}$ where $A_{k}=\prod_{k}^{-1}(0)$ and $B_{k}=\prod_{k}^{-1}(1)$.

By the dimension of $X$ we mean either the covering, small inductive, or large inductive dimension; they are equivalent for separable metric spaces (see [E]). We note that the dimension for a finite-dimensional space $X$ is the largest cardinality of an essential family for $X$.

By a hyperplane in $\mathbf{R}^{k}$ we mean a translate of a linear subspace of $\mathbf{R}^{k}$; the dimension of a hyperplane $H$ is its algebraic dimension, which is the same as its topological dimension. Following the notation from [AK], if $H$ is a hyperplane in $\mathbf{R}^{k}$, then $\widehat{H}$ is the set of translates of $H$. Hyperplanes $H$ and $K$ of dimensions $d_{H}$ and $d_{K}$ are in general position if, for $H^{\prime} \in \widehat{H}$ and $K^{\prime} \in \widehat{K}$, $H^{\prime} \cap K^{\prime} \neq \varnothing$ implies $H^{\prime} \cap K^{\prime}$ is a hyperplane of dimension $\max \left\{0, d_{H}+d_{K}-k\right\}$.

We use $\mathbf{c}$ to denote the cardinality of $\mathbf{R}$, and $\Lambda$ to denote the set of limit ordinals in $\mathbf{c}$.

\section{THE CONSTRUCTION}

Our goal is to construct, given $n, m \geq 2$, and $n \leq d \leq n m-m$, a subset $X$ of $\mathbf{R}^{n}$ with $\operatorname{dim}(X)=n-1, \operatorname{dim}\left(X^{m}\right)=d$, and $\operatorname{dim}\left(X^{\omega}\right)=d$. In order to simplify things, we consider the case where $d=n m-m$. Step 2 below is not necessary in this special case, but is needed for other choices of $d$.

The proof proceeds in five steps:

(1) In $\mathbf{R}^{n m}$ we find a special collection $K$ of $\mathbf{c}$ sets with the property that if $Y \subseteq \mathbf{R}^{n m}$ and $Y \cap k \neq \varnothing$ for each $k \in K$, then $\operatorname{dim}(Y) \geq d$.

(2) In $\mathbf{R}^{n}$ we find a special collection $C$ of $\mathbf{c}$ sets with the property that if $Y \subseteq \mathbf{R}^{n}$ and $Y \cap c \neq \varnothing$ for each $c \in C$, then $\operatorname{dim}(Y) \geq n-1$. This collection was used in $[\mathrm{AK}]$.

(3) For each $q>m$, in $\mathbf{R}^{n q}$ we find families of hyperplanes which give a 
guide to where points in $X^{q}$ will have to fit in $\mathbf{R}^{n q}$. These hyperplanes tell us both how to construct sets in (4) (below) and how we can add new points to a partially formed $X$. The hyperplanes are based on those used in [AK], but are necessarily more complicated in order to allow some growth in dimension.

(4) Since $X^{q}$ is somewhat described by the hyperplanes from (3), we find sets $T_{n q}$ in $\mathbf{R}^{n q}, q>m$ which satisfy $\operatorname{dim}\left(\mathbf{R}^{n q} \backslash T_{n q}\right)=d$, and such that $T_{n q}$ is in "general position" with respect to the hyperplanes from (3).

(5) We construct $X$ inductively so that in the end:

(a) $X^{m}$ intersects each element of $K$ (from (1)),

(b) $X$ intersects each element of $C$ (from (2)), and

(c) $X^{q} \cap T_{n q}=\varnothing$ for all $q>m$.

Then (a) guarantees $\operatorname{dim}\left(X^{m}\right) \geq d$, (b) guarantees $\operatorname{dim}(X) \geq n-1$, and (c) guarantees $\operatorname{dim}\left(X^{q}\right) \leq d$ for all $q>m$, hence $\operatorname{dim}\left(X^{\omega}\right)=d$. Clearly (c) implies that $X$ has empty interior in $\mathbf{R}^{n}$, and this along with (b) gives that $\operatorname{dim}(X)=n-1$.

We proceed.

Step 1. Let $\left\{\left(A_{i}, B_{i}\right): i \in n m\right\}$ be the standard essential family for $\mathbf{I}^{n m} \subseteq \mathbf{R}^{n m}$. Let $l=\{n j-1: 1 \leq j \leq m\}$, and let

$$
K=\left\{\bigcap_{i \in n m \backslash l} c_{i}: c_{i} \text { is a separator of }\left(A_{i}, B_{i}\right)\right\} .
$$

Then we claim:

(1) If $Y \subseteq \mathbf{R}^{n m}, Y \cap k \neq \varnothing$ for each $k \in K$, then $\operatorname{dim}(Y) \geq n m-m$.

Proof. Suppose $Z \subseteq \mathbf{R}^{n m}$ with $\operatorname{dim}(Z)<n m-m$; we find $a k \in K$ with $Z \cap k=\varnothing$. Since $\operatorname{dim}(Z)<n m-m, Z$ is a union of $n m-m$ sets of dimension $\leq 0$ (see, for example, [E]). We may write $Z=\bigcup_{i \in n m \backslash l} Z_{i}$ where $\operatorname{dim}\left(Z_{i}\right) \leq 0$, as $|n m \backslash l|=n m-m$. Since $\operatorname{dim}\left(Z_{t}\right) \leq 0$, there is a separator $c_{t}$ of $\left(A_{t}, B_{t}\right)$ with $c_{t} \cap Z_{t}=\varnothing$ (again by [E]). Then $\bigcap_{t \in n m \backslash l} c_{t}$ is an element of $K$ which misses $Z$.

(2) For each $k \in K, \prod_{l}(k)=\prod_{i \in l} \mathbf{I}_{i}$.

Proof. If not, let $k^{\prime}$ be such that $\prod_{l}\left(k^{\prime}\right) \neq \prod_{i \in l} \mathbf{I}_{i}$. Then there is a point $\left(p_{n-1}, p_{2 n-1}, \ldots, p_{n m-1}\right) \in \prod_{i \in l} \mathbf{I}_{i}$ which is not in $\prod_{l}\left(k^{\prime}\right)$, and we may assume none of the coordinates are 0 (since $\prod_{l}\left(k^{\prime}\right)$ is compact).

Then let $c_{t}=\left\{x \in \mathbf{R}^{n m}: x(t)=p_{t}\right\}$ for $t \in l$. Then $\bigcap_{t \in l} c_{t} \cap k^{\prime}=\varnothing$. This is impossible as $\left\{\left(A_{i}, B_{i}\right): i \leq m n\right\}$ is an essential family for $\mathbf{I}^{n m}$.

Step 2. Let $C=\left\{c: c\right.$ is a continuum from $A_{0}$ to $B_{0}$ in $\left.\mathbf{R}^{n}\right\}$. It is well known (and used in $[\mathrm{AK}]$ ) that if $Y \subseteq \mathbf{R}^{n}$ and $Y \cap c \neq \varnothing$ for all $c \in C$, then $\operatorname{dim}(Y) \geq n-1$.

Step 3. Construction of hyperplanes in $\mathbf{R}^{n q}$, for $q>m$. 
Let $P_{q}$ denote the set of ordered partitions of $q$ of cardinality $m+1$. That is, $p \in \stackrel{q}{P_{q}}$ iff $p=\left(p_{0}, \ldots, p_{m}\right)$ where $\bigcup p_{i}=q$, and $p_{i} \cap p_{j}=\varnothing$ if $i \neq j$; we allow $p_{i}=\varnothing$.

Let $x \in \mathbf{R}^{n q}$; then we write $x=\left(x_{0}, x_{1}, \ldots, x_{q-1}\right)$ where

$$
x_{i}=\left(x_{i}(0), x_{i}(1), \ldots, x_{i}(n-1)\right) \in \mathbf{R}^{n} .
$$

Fix $p \in P_{q} ; p=\left(p_{0}, \ldots, p_{m}\right)$ and define:

(a) $H_{p_{0}}=\left\{x \in \mathbf{R}^{n q}: x_{j}=0\right.$ if $j \notin p_{0}$ and, for $0 \leq r \leq n-1, x_{j}(r)=x_{t}(r)$ if $\left.j, t \in p_{0}\right\}$,

(b) for $0<i<m$,

$$
\begin{array}{r}
H_{p_{i}}=\left\{x \in \mathbf{R}^{n q}: x_{j}=0 \text { if } j \notin p_{i}, x_{j}(r)=x_{t}(r) \text { if } j, t \in p_{i} \text { for } 0 \leq r<n-1,\right. \\
\text { and } \left.x_{j}(n-1)=0 \text { if } j \in p_{i}\right\} .
\end{array}
$$

Remark. In (a) $H_{p_{0}}$ is a linear subspace of $\mathbf{R}^{n q}$ with linear dimension $n$ or 0 ; in (b) $H_{p_{i}}$ is a linear subspace of $\mathbf{R}^{n q}$ of linear dimension $n-1$ or 0 . Note that $H_{p_{m}}$ is not defined.

Using (a) and (b) we define $H_{p}=H_{p_{0}}+H_{p_{1}}+\cdots+H_{p_{m-1}}$. (This is the direct sum.) $H_{p}$ is a linear subspace of $\mathbf{R}^{n q}$ of linear dimension at most $n+(m-1)(n-1)=n m-m+1$. Since $P_{q}$ is finite, $\mathscr{H}=\left\{H_{p}: p \in P_{q}\right\}$ is a finite set.

Step 4. Using the same method as in $[\mathrm{AK}]$, we can prove the following lemma.

Lemma. Given $q>m$, there are countably many spheres $\left\{S_{i}: i \in \omega\right\}$ in $\mathbf{R}^{n q}$ all of dimension $n q-m(n-1)-1$, with $S_{i} \subseteq H_{i}$ where $H_{i}$ is a hyperplane of dimension $n q-m(n-1)$ in $\mathbf{R}^{n q}$ such that

(i) $\operatorname{dim}\left(\mathbf{R}^{n q} \backslash\left(\bigcup S_{i}\right)\right)=m(n-1)$, and

(ii) $H_{i}$ is in general position with respect to each $H^{\prime} \in \widehat{H}$ for each $H \in \mathscr{H}$.

Corollary. If $H \in \mathscr{H}, H^{\prime} \in \widehat{H}$, and $S_{i} \in\left\{S_{j}\right\}$, then $\left|S_{i} \cap H^{\prime}\right| \leq 2$.

Proof.

$$
\begin{aligned}
& \operatorname{dim}\left(H^{\prime}\right) \leq m n-m+1 \\
& \operatorname{dim}\left(H_{i}\right)=n q-m(n-1) .
\end{aligned}
$$

So since $H^{\prime}, H_{i}$ are in general position,

$$
\begin{aligned}
\operatorname{dim}\left(H_{i} \cap H^{\prime}\right) & \leq \max \{0, n q-m(n-1)+m n-m+1-n q\} \\
& =\max \{0,1\} .
\end{aligned}
$$

As $S_{i} \subseteq H_{i}$, then $S_{i} \cap H^{\prime}=S_{i} \cap H_{i} \cap H^{\prime}$. This is $S_{i}$ intersected with a line, hence is at most 2 points.

Now let $T_{n q}=\bigcup S_{i}$. From (i) of the lemma $\mathbf{R}^{n} \backslash T_{n q}$ is of dimension $d=$ $m(n-1)$. The general position properties are given by the corollary. 
Step 5. Construction of $X$.

Let $D=\{\alpha \in \mathbf{c}: \alpha=l+m k$ for some $l \in \Lambda$ and $k \in \omega\}$.

Then enumerate $K$ by $K=\left\{k_{\alpha}: \alpha \in D\right\}$ and $C$ by $C=\left\{c_{\alpha}: \alpha \in D\right\}$.

Assume $\alpha \in D$ and for all $\gamma \in \alpha \cap D$, we have

$$
\begin{aligned}
& Y_{\gamma}=\left\{y_{\beta}: \beta<\gamma+m\right\} \subseteq \mathbf{R}^{n}, \\
& Z_{\gamma}=\left\{z_{\beta}: \beta \leq \gamma, \beta \in D\right\} \subseteq \mathbf{R}^{n}, \text { and } \\
& X_{\gamma}=Y_{\gamma} \cup Z_{\gamma} \text { satisfying }
\end{aligned}
$$

(i) $z_{\beta} \in c_{\beta}$ for all $\beta \in(\gamma+1) \cap D$,

(ii) for each $\delta \in(\gamma+1) \cap D,\left(y_{\delta}, y_{\delta+1}, \ldots, y_{\delta+m-1}\right) \in k_{\delta}$,

(iii) for all $q>m,\left(X_{\gamma}\right)^{q} \cap T_{n q}=\varnothing$,

(iv) when $\lambda, \gamma \in \alpha \cap D$ and $\lambda<\gamma$, then $Y_{\lambda} \subseteq Y_{\gamma}$ and $Z_{\lambda} \subseteq Z_{\gamma}$.

For convenience, we will not allow $(0,0, \ldots, 0)$ in $X_{\gamma}$.

We must produce $Y_{\alpha}$ and $Z_{\alpha}$ so that (i)-(iv) will be satisfied. Let $S_{\alpha}=$ $\bigcup_{\beta<\alpha} Y_{\beta}, T_{\alpha}=\bigcap_{\beta<\alpha} Z_{\beta}$, and $U_{\alpha}=S_{\alpha} \cup T_{\alpha}$; then for all $q>m,\left(U_{\alpha}\right)^{q} \cap$ $T_{n q}=\varnothing$. We will first find $z_{\alpha}$ so that setting $Z_{\alpha}=T_{\alpha} \cup\left\{z_{\alpha}\right\}$, (i) will be satisfied, and then find $y_{\alpha}, y_{\alpha+1}, \ldots, y_{\alpha+m-1}$ so that setting $Y_{\alpha}=S_{\alpha} \cup$ $\left\{y_{\alpha}, y_{\alpha+1}, \ldots, y_{\alpha+m-1}\right\}$ and $X_{\alpha}=Y_{\alpha} \cup Z_{\alpha}$, (ii)-(iv) will be satisfied.

(1) Finding $Z_{\alpha}$. This part is much the same as in [AK]. For each $q>m$, consider the set $V=\bigcup\left\{\left(U_{\alpha} \cup\{x\}\right)^{q}: x \in \mathbf{R}^{n}\right\} \subseteq \mathbf{R}^{n q}$.

For each $p \in P_{q}$ with $p_{i} \neq \varnothing$ only if $i=0$ or $i=m$, consider the hyperplanes of the form $H_{p}+\sigma$ where $\sigma \in \mathbf{R}^{n q}, \sigma_{i} \in U_{\alpha}$ iff $i \in p_{m}$, and $\sigma_{i}=(0,0, \ldots, 0)$ otherwise.

Each $v \in V$ is contained in such a hyperplane. To see this, let $v=$ $\left(v_{0}, v_{1}, \ldots, v_{q-1}\right)$ be in $\left(U_{\alpha} \cup\{x\}\right)^{q}$, and let $\sigma=\left(\sigma_{0}, \sigma_{1}, \ldots, \sigma_{q-1}\right)$ where $\sigma_{i}=(0,0, \ldots, 0)$ if $v_{i}=x$, and $\sigma_{i}=v_{i}$ if $v_{i} \in U_{\alpha} \backslash\{x\}$. Let $p$ be the ordered partition given by $p_{0}=\left\{i: v_{i}=x\right\}, p_{m}=q \backslash p_{0}$, and otherwise $p_{i}=\varnothing$. Then $v \in H_{p}+\sigma$. Also, there are fewer than $\mathrm{c}$ such hyperplanes because $\left|U_{\alpha}\right|<\mathrm{c}$ and $\left|P_{q}\right|<\omega$.

Each such hyperplane, by the corollary in Step 4, intersects $T_{n q}$ in a countable set. Thus setting $B_{q}=\left\{x \in \mathbf{R}^{n}:\left(U_{\alpha} \cup\{x\}\right)^{q} \cap T_{n q} \neq \varnothing\right\},\left|B_{q}\right|<\mathbf{c}$, so there must be $z_{\alpha} \in\left(\mathbf{R}^{n} \backslash \bigcup_{q>m} B_{q}\right) \cap c_{\alpha}$, because $\left|c_{\alpha}\right|=\mathbf{c}$. Hence (i) is satisfied by making $Z_{\alpha}=T_{\alpha} \cup\left\{z_{\alpha}\right\}$. Note that $\left(U_{\alpha} \cup\left\{z_{\alpha}\right\}\right)^{q} \cap T_{n q}=\varnothing$ for all $q>m$.

(2) Finding $\left\{y_{\alpha}, y_{\alpha+1}, y_{\alpha+2}, \ldots, y_{\alpha+m-1}\right\}$. This is the tricky part. One would like to choose $y_{\alpha}$, then $y_{\alpha+1}$ and so on, but it is possible, for example, that choosing a $y_{\alpha}$ a priori would force any $y_{\alpha+1}, y_{\alpha+2}, \ldots, y_{\alpha+m-1}$ where $\left(y_{\alpha}, y_{\alpha+1}, \ldots, y_{\alpha+m-1}\right) \in k_{\alpha}$ to make $\left(X_{\alpha}\right)^{q}$ intersect some set $T_{n q}$. Thus these points must be chosen simultaneously; actually we choose, in sequence, a coordinate of $y_{\alpha+i}$ which will guarantee when we are done that any choice of the other coordinates will not cause an intersection with $T_{n q}$. That the one coordinate can be chosen rather freely is due to (2) of Step 1. 
Let $U_{\alpha}^{+}=U_{\alpha} \cup\left\{z_{\alpha}\right\}$. Inductively we find $r_{t} \in \mathbf{I}$, for $0 \leq t<m$ such that, at each stage $k$, letting $\widehat{Y}_{\alpha+j}=\left\{y \in \mathbf{R}^{n}: y(n-1)=r_{j}\right\}$ for $0 \leq j<k$ and choosing $y_{\alpha+j}^{\prime} \in \widehat{Y}_{\alpha+j}$ arbitrarily, for all $q>m$,

$$
\left(U_{\alpha}^{+} \cup\left\{y_{\alpha}^{\prime}, y_{\alpha+1}^{\prime}, \ldots, y_{\alpha+k-1}^{\prime}\right\}\right)^{q} \cap T_{n q}=\varnothing .
$$

Assume $t \leq m-1$ and that we have $\left\{r_{\alpha}: \alpha<t\right\}$; we want to find $r_{t}$ so that $(*)$ above is satisfied. We claim that for each $q>m$, there are less than $\mathbf{c}$ translates $\left\{H_{\beta}^{q}\right\}$ of hyperplanes in $\mathbf{R}^{n q}$ of the form $H_{p}$ from Step 3 such that for any choice of $y_{\alpha+k}^{\prime} \in \widehat{Y}_{\alpha+k}, 0 \leq k<t$ and any $\sigma \in \mathbf{R}^{n}$,

$$
\left(U_{\alpha}^{+} \bigcup\left\{\sigma, y_{\alpha}^{\prime}, y_{\alpha+1}^{\prime}, \ldots, y_{\alpha+t-1}^{\prime}\right\}\right)^{q} \subseteq \bigcup\left\{H_{\beta}^{q}\right\} .
$$

To see this, first note that if $\tau=\left(\tau_{0}, \tau_{1}, \ldots, \tau_{q-1}\right)$ is in

$$
\left(U_{\alpha}^{+} \bigcup\left\{\sigma, y_{\alpha}^{\prime}, \ldots, y_{\alpha+t-1}^{\prime}\right\}\right)^{q},
$$

then

(v) $\tau_{i}=\sigma$,

(vi) $\tau_{i} \in U_{\alpha}^{+}$, or

(vii) $\tau_{i}=y_{\alpha+k}^{\prime}$ for some $k$, in which case $\tau_{i}(n-1)=r_{k}$.

Now let $p_{0}=\left\{i \in q: \tau_{i}=\sigma\right\}$ and for $1 \leq i<t$, let $p_{i}=\left\{j \in q: \tau_{j}=\right.$ $\left.y_{\alpha+i-1}^{\prime}\right\}$. (These can be disjointified if necessary.) Then let $p_{m}=q \backslash \bigcup_{0 \leq i<t} p_{i}$ (this is essentially where $\tau_{i}$ is in $U_{\alpha}^{+}$), and otherwise let $p_{i}=\varnothing$. We have an ordered partition $p=\left(p_{0}, p_{1}, \ldots, p_{m}\right)$ of $q$.

Then consider

$$
H_{p}(\delta)=H_{p}+\left(\delta_{0}, \ldots, \delta_{q-1}\right)
$$

where

$$
\begin{array}{ll}
\delta_{i}=(0,0, \ldots, 0) & \text { if } i \in p_{0}, \\
\delta_{i}=\left(0,0, \ldots, 0, r_{k}\right) & \text { if } i \in p_{k+1} \quad(k<t), \\
\delta_{i}=\tau_{i} \in U_{\alpha}^{+} & \text {if } i \in p_{m} .
\end{array}
$$

By the choice of $p$ and $\delta, \tau \in H_{p}(\delta)$. Since there are only finitely many choices for $p$ available, and for each choice of $p$ there are less than $\mathbf{c}$ choices for $\delta$, the collection $\left\{H_{\beta}^{q}\right\}$ can be obtained by taking all such possibilities. As each $H_{p}(\delta)$ is a translate of $H_{p},\left|H_{p}(\delta) \cap T_{n q}\right| \leq \omega$, so setting $B_{\alpha+t}^{q}=\{\sigma \in$ $R^{n}:\left(U_{\alpha}^{+} \cup\left\{\sigma, y_{\alpha}^{\prime}, y_{\alpha+1}^{\prime}, \ldots, y_{\alpha+t-1}^{\prime}\right\}\right)^{q} \cap H \cap T_{n q} \neq \varnothing$ for some $H \in\left\{H_{\beta}^{q}\right\}$ and some choice of $\left.y_{\alpha+k}^{\prime} \in \widehat{Y}_{\alpha+k}, 0 \leq k<t\right\}$ we get $\left|B_{\alpha+t}^{q}\right|<\mathbf{c}$, and $\left|\bigcup_{q>m} B_{\alpha+t}^{q}\right|<$ c; in particular $\mathbf{I} \backslash\left(\bigcup_{q>m} \prod_{n-1}\left(B_{\alpha+t}^{q}\right)\right)$ contains a point, which we call $r_{t}$. It follows that, for all $q>m$, and all choices of $y_{\alpha+i}^{\prime} \in \widehat{Y}_{\alpha+i}$, for $0 \leq i \leq t$ that (*) holds; thus the induction can be completed to get $\left\{r_{0}, \ldots, r_{m-1}\right\}$ such that, for all $q>m$, and $y_{\alpha+k}^{\prime} \in \widehat{Y}_{\alpha+k}$, for $0 \leq k<m$,

$$
\left(U_{\alpha}^{+} \cup\left\{y_{\alpha}^{\prime}, y_{\alpha+1}^{\prime}, \ldots, y_{\alpha+m-1}^{\prime}\right\}\right)^{q} \cap T_{n q}=\varnothing .
$$


Now from (2) of Step 1, we know that $k_{\alpha}$ contains a point $\tau=\left(\tau_{0}, \ldots, \tau_{m-1}\right)$ where $\tau_{i}(n-1)=r_{i}$. As $\tau_{i} \in \widehat{Y}_{\alpha+i}$, setting $y_{\alpha+i}=\tau_{i}$ for $i \in m$, and letting $Y_{\alpha}=S_{\alpha} \cup\left\{y_{\alpha}, y_{\alpha+1}, \ldots, y_{\alpha+m-1}\right\}$, we have that (ii)-(iv) are satisfied. Then setting $X=\bigcup X_{\alpha}$, the conditions (a)-(c) of (5) are satisfied.

\section{ACKNOWLEDGMENT}

We gratefully acknowledge the referee's valuable comments and criticisms.

\section{REFERENCES}

[AK] R. D. Anderson and J. E. Keisler, An example in dimension theory, Proc. Amer. Math. Soc. 18 (1967), 709-713.

[E] R. Engelking, Dimension theory, North-Holland, Amsterdam, 1978.

[K] J. Kulesza, The dimension of products of complete separable metric spaces, Fund. Math. (to appear).

[P] E. Pol, On the dimension of the product of metrizable spaces, Bull. Polska Akad. Nauk 26 (1978), 525-533.

[Po] L. S. Pontrjagin, Sur un hypothese fondamentale de la theorie de la dimension, C. R. Acad. Sci. Paris Sér. I Math. 190 (1930), 1105-1107.

[RSW] L. Rubin, R. Schori, and J. Walsh, New dimension theory techniques for constructing infinite dimensional examples, Gen. Topology Appl. 10 (1979), 93-102.

Department of Mathematical Sciences, George Mason University, Fairfax, Virginia 22030 